\title{
Oral antibiotic therapy for the treatment of infective endocarditis: a systematic review
}

\author{
Awad Al-Omari', D William Cameron ${ }^{2,3,4}$, Craig Lee ${ }^{2,4}$ and Vicente F Corrales-Medina $2,3,45^{*}$
}

\begin{abstract}
Background: The role of oral antibiotic therapy in treating infective endocarditis (IE) is not well established.

Methods: We searched MEDLINE, EMBASE and Scopus for studies in which oral antibiotic therapy was used for the treatment of IE.

Results: Seven observational studies evaluating the use oral beta-lactams (five), oral ciprofloxacin in combination with rifampin (one), and linezolid (one) for the treatment of IE caused by susceptible bacteria reported cure rates between $77 \%$ and $100 \%$. Two other observational studies using aureomycin or sulfonamide, however, had failure rates $>75 \%$. One clinical trial comparing oral amoxicillin versus intravenous ceftriaxone for streptococcal IE reported $100 \%$ cure in both arms but its reporting had serious methodological limitations. One small clinical trial $(n=85)$ comparing oral ciprofloxacin and rifampin versus conventional intravenous antibiotic therapy for uncomplicated right-sided S. aureus IE in intravenous drug users (IVDUs) reported cure rates of $89 \%$ and $90 \%$ in each arm, respectively ( $P=0.9$ ); however, drug toxicities were more common in the latter group (62\% versus 3\%; $P<0.01$ ). Major limitations of this trial were lack of allocation concealment and blinding at the delivery of the study drug(s) and assessment of outcomes.

Conclusion: Reported cure rates for IE treated with oral antibiotic regimens vary widely. The use of oral ciprofloxacin in combination with rifampin for uncomplicated right-sided S. aureus IE in IVDUs is supported by one small clinical trial of relatively good quality and could be considered when conventional IV antibiotic therapy is not possible.
\end{abstract}

Keywords: Endocarditis, Treatment, Outcomes, Oral antibiotic therapy

\section{Background}

The hallmark lesion of IE is the endocardial vegetation, a meshwork of platelets, fibrin, bacteria and inflammatory cells in which bacteria proliferate, invade adjacent tissues, and disseminate as septic emboli [1,2]. So long as bacteria are embedded in vegetations, the ability of the immune system to assist with the eradication of these organisms is greatly impaired $[1,2]$. This characteristic underlies the concept, supported by experimental and clinical observations, that high serum levels of bactericidal antibiotics for prolonged periods of time are needed for curing this infection [1-3]. Before the advent of antibiotic therapy, infective endocarditis (IE) was invariably fatal.

\footnotetext{
* Correspondence: vcorrales@toh.on.ca

2Department of Medicine, The Ottawa Hospital, Ottawa, Ontario, Canada

${ }^{3}$ The Ottawa Hospital Research Institute, Ottawa, Ontario, Canada

Full list of author information is available at the end of the article
}

Antibiotics delivered intravenously achieve rapid therapeutic concentrations in blood and perfused tissues, and they are generally regarded as more potent and reliable than their oral counterparts. For these reasons, intravenous (IV) antibiotics are considered the cornerstone of IE treatment [4]. The recommended duration of IV antibiotic therapy for IE varies depending on the characteristics of the infecting organism and the affected endocardial structure but in no instance it is $<2$ weeks and in most cases it extends beyond 4 weeks [5]. However, there are instances in which the options of effective intravenous antibiotics are limited (patients with multiple allergies, resistant bacteria, etc.) or the maintenance of prolonged intravenous access is not desirable (i.e. active intravenous drug users) or at all feasible (i.e. patient's inability to maintain intravascular access). In these situations, oral antibiotic therapy can be an attractive and convenient alternative. However,

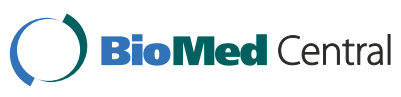

(c) 2014 Al-Omari et al.; licensee BioMed Central Ltd. This is an Open Access article distributed under the terms of the Creative Commons Attribution License (http://creativecommons.org/licenses/by/2.0), which permits unrestricted use, distribution, and reproduction in any medium, provided the original work is properly credited. The Creative Commons Public Domain Dedication waiver (http://creativecommons.org/publicdomain/zero/1.0/) applies to the data made available in this article, unless otherwise stated. 
little is known about the value of this strategy in the setting of IE.

Using a systematic approach, this review examines the literature on the efficacy of oral antibiotic therapy in the treatment of IE.

\section{Methods}

Our review protocol conformed to the Preferred Reporting Items for Systematic Reviews and Meta-Analyses (PRISMA) guidelines [6].

\section{Search strategy}

Our systematic search strategy was developed to capture all articles of IE in which oral antibiotic therapy was used. We included articles reporting in English, French, Spanish and Arabic languages. We searched the following databases: MEDLINE (from 1948 to June 1, 2013), EMBASE (from 1947 to June 1, 2013), and Scopus (from 1960 to June 1, 2013). Reference lists of selected papers were also screened for additional articles of interest. The search strategies used are presented in Additional file 1.

\section{Eligibility criteria}

We only included studies of IE in which the duration of antibiotic treatment was $>2$ weeks and oral antibiotics where the only antibiotics given after 2 weeks of treatment initiation. To be eligible, studies had to a) report mortality and clinical cure as their outcomes of interest; b) report the microbiology of their IE cases; and c) present their data in a way that it allowed for the calculation of outcome rates as a function of the entire study cohort. Studies with focus on culture negative endocarditis were excluded. We also excluded case series (defined as studies with $<10$ participants) and articles without original data.

\section{Selection of studies}

All titles and abstracts of the citations identified by our literature search were independently screened by two investigators (AA-O and VFC-M). Relevant articles were reviewed in their entirety. Each investigator made a recommendation for inclusion or exclusion of single articles and if discordant, a third investigator solved the discrepancy (CL). When 2 or more articles had overlap of their populations and reported on the same outcomes, only the most inclusive article was considered.

\section{Data extraction, synthesis and analyses}

Using a standardized form, we systematically collected data on the outcomes of interest, the characteristics of the populations studied, whether IE involved the right or left heart valves, and several aspects of the study setting and methodological design. For purposes of this review, cure was defined as both microbiological and clinical resolution of the infection. We used the McMaster University literature appraisal recommendations to evaluate the quality of observational studies [7]; whereas for clinical trials, we used the Consolidated Standards of Reporting Trials (CONSORT) guidelines [8]. We made every effort to calculate pooled incidence rates for the outcomes of interest when feasible.

\section{Results}

Of a total of 709 titles retrieved by our search strategy, 25 articles were considered for review based on their title and abstract. Hereafter, 14 more articles were excluded based on exclusion criteria (Additional file 2), leaving 11 studies for the final analyses [9-19] (Figure 1). Of these, 9 were observational [9-17] and 2 were randomized controlled trials (RCTs) [18,19]. Tables 1 and 2 summarize the characteristics of the selected studies.

\section{Observational studies}

All observational studies involved patients that were hospitalized at the moment of enrolment. Three (33\%) of these studies were of prospective design [10-12] and 6 (67\%) were retrospective [9,13-17]. Two (22\%) studies focused on left- sided IE $[9,12]$, one in right-sided IE (11\%) [10], and six (60\%) did not specify this information [11,13-17]. One study (11\%) involved only intravenous drug users (IVDUs) [10] and one (11\%) involved only paediatric cases [13] (Table 1).

\section{Quality assessment}

Only two studies (22\%) established a uniform process for patient enrolment $[9,10]$. One study $(11 \%)$ relied on modified Duke's criteria for the diagnosis of IE [9]; two studies $(22 \%)$ used a combination of suggestive clinical findings, echocardiographic findings and positive blood cultures [10,11]; four studies $(44 \%)$ made this diagnosis based of the pre-existence of valvular disease and the presence of suggestive clinical findings and/or positive blood cultures [12,13,15,16]; and two studies $(22 \%)$ did not provide case definitions $[14,17]$. Only three studies specified inclusion and exclusion criteria [9-11]. No study provided comparative information for eligible patients that were not ultimately enrolled in the study or did comparative analysis of outcomes relative to a control group. All studies gave information of outcomes for all patients enrolled and provided enough information to identify whether the institution in which the investigation was carried out was a referral center or not. The individual quality performance of each of these studies is summarized in Table 3.

\section{Therapy and outcomes}

Four studies (44\%) used oral antibiotic therapy for the entire duration of treatment $[11,14,16,17]$, two $(22 \%)$ used 


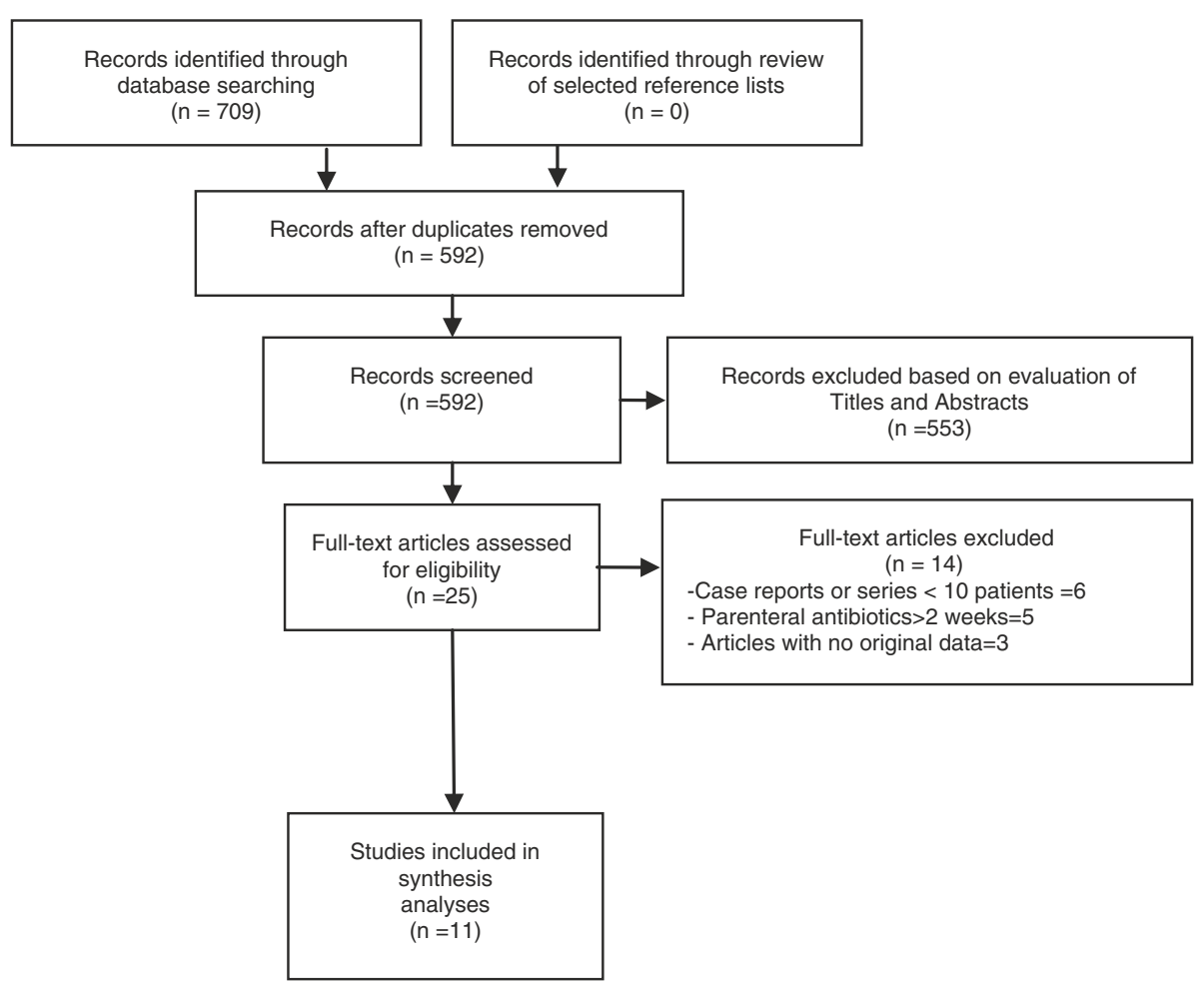

Figure 1 Flow diagram of the process for the selection of articles included in this review.

oral therapy along with adjuvant parenteral streptomycin for the first 2 weeks of treatment $[12,15]$, and three $(33 \%)$ used oral therapy only after an initial short course $(<2$ weeks) of IV antibiotics $[9,10,13]$. Oral therapies included beta-lactams (amoxicillin, penicillin $\mathrm{V}$, ampicillin, cloxacillin and dicloxacillin) - with or without probenecid - in five (56\%) studies [11-15]; fluoroquinolones (ciprofloxacin) in combination with rifampin in one (16\%) [10]; and sulfonamide, aureomycin and linezolid in one study each $[9,16,17]$.

All studies described the microbiological aetiology of their cases. Seven studies (78\%) involved patients with infections caused primarily by Streptococcus sp. [11-17], while two (22\%) included mainly cases of S. aureus disease $[9,10]$. All but one study [17] reported antibiotic susceptibility of the etiologic bacteria.

The follow-up time widely varied among reports (4 weeks to 15 years). Seven studies (78\%) reported cure rates between 77-100\% [9-15]. Two other studies, however, had failure rates of $90 \%$ [17] and 74\% [16]. The former of these involved cases of S. viridians IE treated with oral sulfonamide [17]; whereas the latter used oral aureomycin to treat $S$. viridans (55\%), enterococcus (18\%), or culture negative (27\%) IE [16] (Table 1).

\section{Clinical trials}

We identified 2 clinical trials. Heldman et al [18] focused on right-sided IE caused by $S$. aureus in IVDUs whereas
Stamboulian et al [19] studied left-sided IE caused by Streptococcus sp. (S. viridians $50 \%$ and S. bovis 50\%) (Table 2).

\section{Quality assessment}

Both studies used a combination of suggestive clinical signs, positive blood cultures and echocardiographic findings in their case definition of IE $[18,19]$. Both studies also defined eligibility criteria, provided details of the setting and location of the study, and gave a detailed description of the intervention used. Only Heldman et al [18] detailed the processes followed for ascertainment and measurement of clinical outcomes, established adequate sequence generation for randomization, analyzed data on the safety of the intervention, described the participants' flow in the study, addressed concerns for incomplete outcome data and risk of selective reporting, and provided information on the accessibility to their study protocol, trial registration and the funding for their work. No study complied with concealment or blinding of case allocation at the delivery of the study drug(s) or assessment of outcomes. The individual quality performance of each of these studies is summarized in Table 4.

\section{Therapy used and outcomes}

Heldman et al [18] compared 4-week courses of IV vancomycin or oxacillin (with gentamicin for the first 
Table 1 Observational studies of oral antibiotic therapy for infective endocarditis

\begin{tabular}{|c|c|c|c|c|c|c|c|}
\hline Reference & Cases & Design & Case definition & Microbiology & $\begin{array}{l}\text { Assessment } \\
\text { of antibiotic } \\
\text { susceptibility }\end{array}$ & Therapy & Cure \\
\hline \multirow[t]{3}{*}{ Colli et al, Italy [9] } & \multirow{3}{*}{$\begin{array}{l}12 \text { NVIE and } 2 \\
\text { PVIE requiring } \\
\text { acute valve } \\
\text { replacement(all } \\
\text { left-sided) }\end{array}$} & \multirow{3}{*}{$\begin{array}{l}\text { Retrospective. } \\
\text { Mean follow- } \\
\text { up was } 20.8 \pm \\
7 \text { months }\end{array}$} & \multirow[t]{3}{*}{ By Duke criteria } & MRSA (60\%) & \multirow[t]{3}{*}{ Yes } & \multirow{3}{*}{$\begin{array}{l}\text { IV vancomycin for } \\
5.3 \pm 3.4 \text { days } \\
\text { followed by oral } \\
\text { linezolid for } \\
3 \text { weeks }\end{array}$} & \multirow[t]{3}{*}{$100 \%$} \\
\hline & & & & S. viridans (30\%) & & & \\
\hline & & & & $\begin{array}{l}\text { Enterococcus } \\
\text { sp. }(10 \%)\end{array}$ & & & \\
\hline Dworkin et al, USA [10] & $\begin{array}{l}13 \text { IVDUs with } \\
\text { NVIE (all right-sided } \\
\text { with no systemic } \\
\text { metastasis) }\end{array}$ & $\begin{array}{l}\text { Prospective. } \\
\text { 4-week follow-up }\end{array}$ & $\begin{array}{l}\geq 2 \text { positive blood } \\
\text { cultures AND any of } \\
\text { the following: Vegetations } \\
\text { on echocardiogram } \\
\text { (definite }-3 \text { cases) OR } \\
\text { pulmonary infiltrates/ } \\
\text { effusion or tricuspid } \\
\text { insufficiency murmur } \\
\text { (probable - } 6 \text { cases) } \\
\text { OR no other identifiable } \\
\text { source for the infection } \\
\text { (possible - } 1 \text { case) }\end{array}$ & S. aureus (100\%) & Yes & $\begin{array}{l}\text { IV ciprofloxacin and } \\
\text { oral rifampin for } 1 \\
\text { week followed by } \\
\text { oral ciprofloxacin } \\
\text { and oral rifampin } \\
\text { for } 3 \text { weeks }\end{array}$ & $77 \%$ \\
\hline \multirow[t]{2}{*}{$\begin{array}{l}\text { Chetty et al, South } \\
\text { Africa [11] }\end{array}$} & \multirow{2}{*}{$\begin{array}{l}15 \text { NVIE (right-sided } \\
\text { vs. left-sided not } \\
\text { specified, all cases } \\
\text { were considered } \\
\text { uncomplicated) }\end{array}$} & \multirow[t]{2}{*}{$\begin{array}{l}\text { Prospective. } \\
\text { 3-year follow-up }\end{array}$} & \multirow{2}{*}{$\begin{array}{l}\text { Characteristics clinical } \\
\text { features AND any of } \\
\text { the following: Positive } \\
\text { blood cultures OR } \\
\text { vegetations on } \\
\text { echocardiogram }\end{array}$} & $\begin{array}{l}\text { Streptococcus } \\
\text { sp. }(60 \%)\end{array}$ & \multirow[t]{2}{*}{ Yes } & \multirow{2}{*}{$\begin{array}{l}\text { High dose oral } \\
\text { amoxycillin for } \\
6 \text { weeks ( } 47 \% \\
\text { also received } \\
\text { probenecid) }\end{array}$} & \multirow[t]{2}{*}{$87 \%$} \\
\hline & & & & Culture negative (40\%) & & & \\
\hline Pinchas et al, Israel [12] & $\begin{array}{l}11 \text { NVIE (all left-sided, } \\
\text { considered } \\
\text { uncomplicated) }\end{array}$ & $\begin{array}{l}\text { Prospective. } \\
\text { Follow-up varied } \\
\text { from } 3 \text { months } \\
\text { to } 12 \text { years }\end{array}$ & $\begin{array}{l}\text { Fever AND pre-existing } \\
\text { valvular heart disease } \\
\text { AND multiple positive } \\
\text { blood cultures }\end{array}$ & $\begin{array}{l}\text { S. viridans } \\
(100 \%)\end{array}$ & Yes & $\begin{array}{l}\text { High dose oral } \\
\text { ampicillin for } 6 \\
\text { weeks with } \\
\text { probenecid for } \\
\text { the first } 4 \text { weeks. } \\
\text { IM streptomycin } \\
\text { for the first } 2 \text { weeks }\end{array}$ & $90 \%$ \\
\hline \multirow[t]{2}{*}{ Phillips et al, UK [13] } & \multirow{2}{*}{$\begin{array}{l}13 \text { NVIE (right-sided } \\
\text { vs. left-sided not } \\
\text { specified) - all } \\
\text { children }\end{array}$} & \multirow[t]{2}{*}{$\begin{array}{l}\text { Retrospective. } \\
\text { Follow-up varied } \\
\text { from 1-15 years }\end{array}$} & \multirow{2}{*}{$\begin{array}{l}\text { Pre-existing valvular } \\
\text { disease AND characteristic } \\
\text { clinical features AND positive } \\
\text { blood cultures }\end{array}$} & $\begin{array}{l}\text { S, viridans }(62 \%) \\
\text { Staphylococcus } \\
\text { sp. }(23 \%)\end{array}$ & \multirow[t]{2}{*}{ Yes } & \multirow{2}{*}{$\begin{array}{l}\text { IV therapy for }<2 \\
\text { weeks ( } 92 \% \leq 3 \text { days) } \\
\text { followed by oral } \\
\text { penicillin V, ampicillin, } \\
\text { cloxacillin, flucloxacillin } \\
\text { or erythromycin for } \\
6-8 \text { weeks }\end{array}$} & \multirow[t]{2}{*}{$100 \%$} \\
\hline & & & & $\begin{array}{l}\text { Other streptococci } \\
\text { or Enterococcus sp. (15\%) }\end{array}$ & & & \\
\hline \multirow[t]{3}{*}{ Gray et al, UK [14] } & \multirow{3}{*}{$\begin{array}{l}13 \text { NVIE (right-sided } \\
\text { vs. left-sided not } \\
\text { specified) }\end{array}$} & \multirow{3}{*}{$\begin{array}{l}\text { Retrospective. } \\
\text { 3-month } \\
\text { follow-up }\end{array}$} & \multirow[t]{3}{*}{ Not specified } & $\begin{array}{l}\text { S. viridans. } \\
(62 \%)\end{array}$ & \multirow[t]{3}{*}{ Yes } & \multirow{3}{*}{$\begin{array}{l}\text { Oral ampicillin or } \\
\text { propicillin (with or } \\
\text { without probenecid) } \\
\text { for } 6 \text { weeks }\end{array}$} & \multirow[t]{3}{*}{$92 \%$} \\
\hline & & & & E. faecalis (1\%) & & & \\
\hline & & & & $\begin{array}{l}\text { Culture } \\
\text { negative (37\%) }\end{array}$ & & & \\
\hline
\end{tabular}


Table 1 Observational studies of oral antibiotic therapy for infective endocarditis (Continued)

\begin{tabular}{|c|c|c|c|c|c|c|c|}
\hline \multirow[t]{3}{*}{ Campeau et al, Canada [15] } & \multirow{3}{*}{$\begin{array}{l}10 \text { NVIE (right-sided } \\
\text { vs. left-sided not } \\
\text { specified) }\end{array}$} & \multirow{3}{*}{$\begin{array}{l}\text { Retrospective. } \\
\text { Follow-up varied } \\
\text { from 6-30 } \\
\text { months }\end{array}$} & \multirow{3}{*}{$\begin{array}{l}\text { Pre-existing valvular } \\
\text { disease AND Characteristic } \\
\text { clinical features AND } \geq 2 \\
\text { positive blood cultures }\end{array}$} & S. viridans (60\%) & \multirow[t]{3}{*}{ Yes } & \multirow{3}{*}{$\begin{array}{l}\text { Oral phenithicillin for } \\
4-6 \text { weeks (IM } \\
\text { streptomycin for } \\
\text { the first } 2 \text { weeks in } \\
6 \text { cases, concomitant } \\
\text { probenecid in } 2 \text { cases) }\end{array}$} & \multirow[t]{3}{*}{$80 \%$} \\
\hline & & & & E. faecalis (30\%) & & & \\
\hline & & & & $\begin{array}{l}\text { Anaerobic } \\
\text { bacteria (10\%) }\end{array}$ & & & \\
\hline \multirow[t]{3}{*}{ Friedberg et al, USA [16] } & \multirow{3}{*}{$\begin{array}{l}11 \text { NVIE (right-sided } \\
\text { vs. left-sided not } \\
\text { specified) }\end{array}$} & \multirow{3}{*}{$\begin{array}{l}\text { Retrospective. } \\
\text { Follow-up not } \\
\text { specified }\end{array}$} & \multirow{3}{*}{$\begin{array}{l}\text { Pre-existing rheumatic } \\
\text { valvular disease AND } \\
\text { Unexplained fever for } \\
\geq 2 \frac{1}{2} \text { weeks }\end{array}$} & S. viridans (55\%) & \multirow[t]{3}{*}{ Yes } & \multirow{3}{*}{$\begin{array}{l}\text { Oral Aureomycin for } \\
5-8 \text { weeks }\end{array}$} & \multirow[t]{3}{*}{$36 \%$} \\
\hline & & & & E. faecalis (18\%) & & & \\
\hline & & & & $\begin{array}{l}\text { Culture } \\
\text { negative (27\%) }\end{array}$ & & & \\
\hline \multirow[t]{4}{*}{ Schein et al, USA [17] } & \multirow{4}{*}{$\begin{array}{l}81 \text { NVIE (right-side } \\
\text { vs. left-sided not } \\
\text { specified) }\end{array}$} & \multirow{4}{*}{$\begin{array}{l}\text { Retrospective. } \\
\text { Follow-up varied } \\
\text { from 2-8 years }\end{array}$} & \multirow[t]{4}{*}{ Not specified } & $\begin{array}{l}\text { Streptococcus } \\
\text { sp. }(94 \%)\end{array}$ & \multirow[t]{4}{*}{ Not specified } & \multirow{4}{*}{$\begin{array}{l}\text { Oral sulfonamides } \\
\text { (sulfanilamide, } \\
\text { sulfapyridine, } \\
\text { sulfathiazole or } \\
\text { sulfadiazine) for } \\
10 \text { days-14 weeks }\end{array}$} & \multirow[t]{4}{*}{$10 \%$} \\
\hline & & & & S. aureus (1\%) & & & \\
\hline & & & & $\begin{array}{l}\text { Enterococcus } \\
\text { sp. }(1 \%)\end{array}$ & & & \\
\hline & & & & H. influenza (4\%) & & & \\
\hline
\end{tabular}

NVIE denotes cases of native valve infective endocarditis. PVIE denotes cases of prosthetic valve infective endocarditis. IV denotes intravenous. IVDUs denotes intravenous drug users. MSSA denotes methicillin-sensitive S. aureus. MRSA denotes methicillin-resistant S. aureus. CoNS denotes coagulase-negative staphylococcus. GNB denotes gram-negative bacilli. Unless specified otherwise, all cohorts were primarily of adult patients. All reports reported follow-up $\geq 3$ months. 
Table $\mathbf{2}$ Clinical trials of oral antibiotic therapy for infective endocarditis

\begin{tabular}{|c|c|c|c|c|c|c|}
\hline Reference & Cases & Design & Case definition & Microbiology & Therapy & Results \\
\hline \multirow[t]{2}{*}{$\begin{array}{l}\text { Heldman et al, } \\
\text { USA [18] }\end{array}$} & \multirow{2}{*}{$\begin{array}{l}85 \text { IVDUs with NVIE } \\
\text { (all right-sided with } \\
\text { no systemic } \\
\text { metastases), } 40 \text { in } \\
\text { the oral therapy } \\
\text { arm and } 45 \text { in the } \\
\text { IV therapy arm }\end{array}$} & \multirow{2}{*}{$\begin{array}{l}\text { Prospective, } \\
\text { randomized, } \\
\text { open label. } \\
\text { 1-month } \\
\text { follow-up }\end{array}$} & \multirow{2}{*}{$\begin{array}{l}-\geq 2 \text { positive blood } \\
\text { cultures AND any of } \\
\text { the following: Valvular } \\
\text { vegetations on } \\
\text { echocardiogram (definite - } \\
15 \text { cases) OR evidence } \\
\text { of pulmonary emboli } \\
\text { on chest X-ray or tricuspid } \\
\text { insufficiency murmur } \\
\text { (probable - } 26 \text { cases) OR } \\
\text { no other identifiable source } \\
\text { for the infection (possible - } \\
44 \text { cases) }\end{array}$} & \multirow[t]{2}{*}{$\begin{array}{l}\text { MRSA (5\%) } \\
\text { MSSA (89\%) } \\
\text { CoNS (6\%) }\end{array}$} & \multirow{2}{*}{$\begin{array}{l}\text { Oral ciprofloxacin } \\
\text { and rifampin for } 4 \\
\text { weeks vs. IV oxacillin } \\
\text { or vancomycin (IV } \\
\text { gentamicin for the first } \\
5 \text { days) for } 4 \text { weeks }\end{array}$} & $\begin{array}{l}\text { Cure rate: } 90 \% \text { (oral } \\
\text { therapy) vs. } 91 \% \text { (IV } \\
\text { therapy), } p=0.9\end{array}$ \\
\hline & & & & & & $\begin{array}{l}\text { Treatment toxicity: } \\
3 \% \text { (oral therapy) } \\
\text { vs. } 62 \% \text { (IV therapy), } \\
p<0.001\end{array}$ \\
\hline \multirow{2}{*}{$\begin{array}{l}\text { Stamboulian } \\
\text { et al, Argentine } \\
\text { [19] }\end{array}$} & \multirow[t]{2}{*}{$\begin{array}{l}30 \text { NVIE (all left-sided), } \\
15 \text { in each arm }\end{array}$} & \multirow{2}{*}{$\begin{array}{l}\text { Prospective, } \\
\text { randomized, } \\
\text { open label. } 3 \\
\text { to 6-motnh } \\
\text { follow-up }\end{array}$} & \multirow{2}{*}{$\begin{array}{l}-\geq 2 \text { positive blood cultures } \\
\text { AND any of the following: } \\
\text { New or changing regurgitant } \\
\text { murmur OR predisposing } \\
\text { heart disease OR vascular } \\
\text { phenomena OR valvular } \\
\text { vegetation on echocardiogram }\end{array}$} & $\begin{array}{l}\text { S. viridans } \\
(50 \%)\end{array}$ & \multirow{2}{*}{$\begin{array}{l}\text { IV or IM ceftriaxone } \\
\text { for } 2 \text { weeks followed } \\
\text { by high dose oral } \\
\text { amoxicillin for } 2 \text { weeks } \\
\text { vs. IV or IM ceftriaxone } \\
\text { for } 4 \text { weeks }\end{array}$} & \multirow{2}{*}{$\begin{array}{l}\text { Cure rate: } 100 \% \\
\text { in both arms. } \\
\text { Treatment toxicity } \\
\text { not reported }\end{array}$} \\
\hline & & & & $\begin{array}{l}\text { S. bovis } \\
(50 \%)\end{array}$ & & \\
\hline
\end{tabular}

NVIE denotes cases of native valve infective endocarditis. IV denotes intravenous. IM denotes intramuscular. IVDUs denotes intravenous drug users. MSSA denotes methicillin-sensitive S. aureus. MRSA denotes methicillin-resistant S. aureus. CoNS denotes coagulase-negative staphylococcus. All reports reported follow-up $\geq 2$ months.

five days) vs. oral ciprofloxacin plus rifampin. The cure rates with oral and IV therapy were $89 \%$ and $90 \%$, respectively $(P=0.9)$. However, drug toxicity was observed in only 1 patient $(2.8 \%)$ in the oral therapy arm versus $24(61.5 \%)$ in the IV therapy arm $(P<0.01)$. Adverse events involved hepatotoxicity (1 patient in the oral therapy arm and in 13 patients in the IV therapy arm), and nephrotoxicity (10 patients in the IV therapy arm).

Stamboulian et al [19] compared a 4-week course of IV ceftriaxone versus 2 weeks of IV ceftriaxone followed by 2 weeks of oral amoxicillin (1 gm four times daily). They reported $100 \%$ cure rate in both arms after a follow up of six months (Table 2).

\section{Discussion}

The main findings of our review are: a) Reported cure rates for IE caused by susceptible organisms and treated with appropriate oral antibiotic regimens range between 77-100\%; and b) Limited evidence from one small clinical trial suggests that the combination of oral ciprofloxacin and rifampin for the treatment of uncomplicated right-sided IE caused by susceptible strains of $S$. aureus in IVDUs could be as effective as, and produce less adverse events than conventional IV antibiotic regimens.

Previous narrative reviews advocated a limited role for oral antibiotics in the treatment of IE [4]. These opinions, however, were largely based on theoretical considerations

Table 3 Quality assessment of observational studies of oral antibiotic therapy in infective endocarditis

\begin{tabular}{|c|c|c|c|c|c|c|c|}
\hline & $\begin{array}{l}\text { Establishes } \\
\text { uniform } \\
\text { process } \\
\text { for patient } \\
\text { enrolment }\end{array}$ & $\begin{array}{l}\text { Provides } r \\
\text { case-definition } \\
\text { for infective } \\
\text { endocarditis }\end{array}$ & $\begin{array}{l}\text { Provides } \\
\text { criteria for } \\
\text { inclusion and } \\
\text { exclusion }\end{array}$ & $\begin{array}{l}\text { Provides comparative } \\
\text { information for } \\
\text { eligible patients } \\
\text { ultimately not } \\
\text { enrolled }\end{array}$ & $\begin{array}{l}\text { Provides } \\
\text { Comparative } \\
\text { analysis with a } \\
\text { control group }\end{array}$ & $\begin{array}{l}\text { All enrolled } \\
\text { patients } \\
\text { accounted for } \\
\text { in the results? }\end{array}$ & $\begin{array}{l}\text { It is possible } \\
\text { to determine } \\
\text { whether the } \\
\text { study institutions } \\
\text { were referral } \\
\text { centers }\end{array}$ \\
\hline Colli et al [9] & $\sqrt{ }$ & $\sqrt{ }$ & $\sqrt{ }$ & $\mathrm{x}$ & $\mathrm{x}$ & $\sqrt{ }$ & $\sqrt{ }$ \\
\hline Dworkin et al [10] & $\sqrt{ }$ & $\sqrt{ }$ & $\sqrt{ }$ & $x$ & $x$ & $\sqrt{ }$ & $\sqrt{ }$ \\
\hline Chetty et al [11] & $x$ & $\sqrt{ }$ & $\sqrt{ }$ & $\mathrm{x}$ & $\mathrm{x}$ & $\sqrt{ }$ & $\sqrt{ }$ \\
\hline Pinchas et al [12] & $x$ & $\sqrt{ }$ & $x$ & $x$ & $x$ & $\sqrt{ }$ & $\sqrt{ }$ \\
\hline Phillips et al [13] & $\mathrm{x}$ & $\sqrt{ }$ & $x$ & $\mathrm{x}$ & $\mathrm{x}$ & $\sqrt{ }$ & $\sqrt{ }$ \\
\hline Gray et al [14] & $x$ & $x$ & $x$ & $x$ & $x$ & $\sqrt{ }$ & $\sqrt{ }$ \\
\hline Campeau et al [15] & $x$ & $\sqrt{ }$ & $x$ & $\mathrm{x}$ & $\mathrm{x}$ & $\sqrt{ }$ & $\sqrt{ }$ \\
\hline Friedberg et al [16] & $x$ & $\sqrt{ }$ & $x$ & $\mathrm{x}$ & $x$ & $\sqrt{ }$ & $\sqrt{ }$ \\
\hline Schein et al [17] & $x$ & $x$ & $x$ & $x$ & $x$ & $\sqrt{ }$ & $\sqrt{ }$ \\
\hline
\end{tabular}


Table 4 Quality assessment of clinical trials of oral antibiotic therapy in infective endocarditis

\begin{tabular}{|c|c|c|c|c|c|c|c|c|c|c|c|c|c|c|}
\hline $\begin{array}{l}\text { Provides } \\
\text { case definition } \\
\text { for infective } \\
\text { endocarditis }\end{array}$ & $\begin{array}{l}\text { Defines } \\
\text { eligibility } \\
\text { criteria }\end{array}$ & $\begin{array}{l}\text { Provides } \\
\text { details of } \\
\text { the setting } \\
\text { and location } \\
\text { of the study }\end{array}$ & $\begin{array}{l}\text { Provides } \\
\text { detailed } \\
\text { description } \\
\text { of the } \\
\text { intervention }\end{array}$ & $\begin{array}{l}\text { Details } \\
\text { processes for } \\
\text { ascertainment } \\
\text { and measurement } \\
\text { of outcomes }\end{array}$ & $\begin{array}{l}\text { Provides } \\
\text { justification } \\
\text { for sample } \\
\text { size }\end{array}$ & $\begin{array}{l}\text { Establishes } \\
\text { an adequate } \\
\text { sequence } \\
\text { generation }\end{array}$ & $\begin{array}{l}\text { Allocation } \\
\text { concealment }\end{array}$ & $\begin{array}{l}\text { Blinding } \\
\text { (study } \\
\text { drug) }\end{array}$ & $\begin{array}{l}\text { Blinding } \\
\text { (outcomes) }\end{array}$ & $\begin{array}{l}\text { Analysis } \\
\text { of safety } \\
\text { of the } \\
\text { intervention }\end{array}$ & $\begin{array}{l}\text { Describes } \\
\text { participants' } \\
\text { flow }\end{array}$ & $\begin{array}{l}\text { Addresses } \\
\text { incompleted } \\
\text { outcome } \\
\text { data }\end{array}$ & $\begin{array}{l}\text { Addresses } \\
\text { risk of } \\
\text { selective } \\
\text { reporting }\end{array}$ & $\begin{array}{l}\text { Provides } \\
\text { information } \\
\text { on accessibility } \\
\text { to the study } \\
\text { protocol, trial } \\
\text { registration } \\
\text { and funding } \\
\text { for the study }\end{array}$ \\
\hline
\end{tabular}

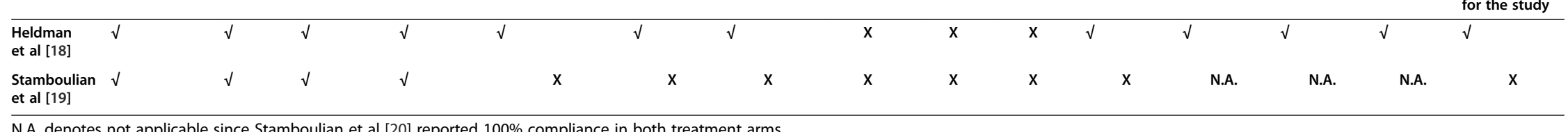

N.A. denotes not applicable since Stamboulian et al [20] reported 100\% compliance in both treatment arms. 
and anecdotal experience [4]. In contrast, this study is the first systematic analysis of the existing published evidence on this topic.

The success of antibiotics in controlling bacterial growth and replication is dependent on: a) The susceptibility of the pathogen to the anti-infective that is used; b) The pharmacokinetics of this drug (i.e. whether its bioavailability and distribution allow it to reach the site of infection in sufficient concentration); and, c) Appropriate duration of therapy (especially when the 2 previous criteria are not fully met). Although the first available formulations of antibiotics had unpredictable absorption when given orally, more stable and absorbable compounds soon became available [4]. Oral betalactams used in the studies of this review included, among others, ampicillin, amoxicillin, phenithicillin and penicillin V [11-15]. Although the pharmacokinetic profile of oral ampicillin is known to be suboptimal [20], the studies in which this antibiotic was used reported high response rates [12-14]. This is likely explained by the fact that the organisms causing IE in those series were mainly streptococci (which remain highly sensitive to beta-lactams to this date - penicillin MIC $\leq 0.12$ ), and that large doses of oral ampicillin were used, frequently supplemented with probenecid $[12,14]$. Oral amoxicillin, on the other hand, has excellent bioavailability (>90\%) and low binding to serum proteins (17\%), which maximizes its tissue penetration [21]. Typical doses of oral amoxicillin (1 g q8h) produce peak and 6-hour serum concentrations of $16 \mathrm{ug} / \mathrm{ml}$ and $1.1 \mathrm{ug} / \mathrm{ml}$, respectively $[4,22,23]$. Further, adding probenecid $1 \mathrm{~g}$ to each dose of amoxicillin increases its peak and trough serum concentrations by $30 \%$ and 4 -fold, respectively [22]. In our review, we found only one observational study reporting $80 \%$ cure rate with oral amoxicillin in 15 cases of IE caused mainly by susceptible streptococci, and one poorquality small clinical trial $(\mathrm{n}=30)$ in which the use of high-dose oral amoxicillin for 2 weeks after 2 initial weeks of IV ceftriaxone resulted in the cure of all patients in the treatment arm [11,19]. Therefore, while pharmacological considerations make oral amoxicillin a plausible alternative for the treatment of IE caused by susceptible bacteria, the clinical evidence supporting this approach is still not robust. However, because streptococci continue to be a leading cause of IE (40\% - 60\% of native valve endocarditis in the community setting) [1] and oral amoxicillin is inexpensive and widely available, this therapeutic approach should be further investigated in adequately designed clinical trials. Beacause oral penicillin $\mathrm{V}$ and phenithicillin also have favourable pharmacokinetic profiles, the same considerations apply to these drugs [24-26].

$S$. aureus is the second most prominent cause of community acquired IE and the leading cause among those who acquired the infection in healthcare settings and among IVDUs [2]. Ciprofloxacin has bactericidal activity against $S$. aureus and a favourable pharmacokinetic profile when given orally $(70 \%$ bioavailability and serum protein binding rate of $30 \%$ ), but the emergence of resistance during treatment of $S$. aureus experimental disease is well described [27,28]. Similarly, rifampin is bactericidal against S.aureus, has almost complete oral bioavailability, and shows little binding to serum proteins; however, it also has a low threshold for the development of spontaneous resistance during therapy [29]. Although combining both agents has unpredictable effects in their anti-bacterial activity in vitro (i.e. synergistic versus antagonistic), it consistently reduces the development of resistance to either drug [30,31]. We identified one prospective observational study [10] and one randomized clinical trial [18] in which the combination of oral ciprofloxacin and rifampin proved not only effective against uncomplicated $S$. aureus right-sided IE in IVDUs but, in the latter case, it was better tolerated than conventional IV therapy. However, the methodological limitations of these studies (Tables 3 and 4) warrant confirmatory investigations before this approach could be widely adopted. In the meantime, this antibiotic combination regimen should only be used in selected cases for which currently favored IV regimens (including beta-lactams or glycopeptides) are not suitable. Notably, development of resistance to the combination of ciprofloxacin and rifampin has been reported in at least one human case of IE [32]. Newer fluoroquinolones such as levofloxacin and moxifloxacin also have favourable pharmacologic profile when given orally and are bactericidal against $S$. aureus, and in contrast to ciprofloxacin, the development of in-vivo resistance appears rare $[27,33,34]$. Both levofloxacin and moxifloxacin have also proved effective in animal models of infective endocarditis $[35,36]$ and in anecdotal human cases $[37,38]$. Therefore, it would also be reasonable to consider the oral formulations of these drugs in future studies for the treatment of this infection. We found that clinical experience with the use of oral anti-staphylococcal penicillins such as cloxacillin and flucloxacillin (used in one report of our review) [13] for the treatment of S. aureus IE is very limited and therefore, this approach should only be considered in controlled research settings.

Other oral antibiotics used in the reports identified by our review include linezolid, aureomycin, sulphonamide, and erythromycin $\quad[9,10,13,17]$. Oral linezolid has excellent pharmacologic profile (bioavailability $>99 \%$ and serum protein binding rate $30 \%$ ) and there is a growing body of evidence of its efficacy in serious infections caused by Gram-positive cocci [39]. The promising results with the use of oral linezolid for the treatment IE reported by Colli et al [9] warrant further confirmation 
in clinical trials. Aureomycin (chlortetracyclin) is an old tetracycline derivative with almost complete GI absorption that is no longer available for clinical use [40]. Aureomycin was only $36 \%$ effective in a small series of 11 patients with IE mainly due to E. faecalis and strepcocci [16]. However, newer tetracyclines such as minocycline and doxycycline (which also have excellent bioavailability and are active against gram positive organisms) have more recently been proposed as potential oral alternatives for treatment of IE caused by common bacteria [4]. Doxycycline has long been used for treatment of Q fever IE $[41,42]$ but its unpredictable efficacy against S.aureus would limit its use in other IE settings. Minocycline, on the other hand, has consistent and reliable activity against gram-positive organisms, including $S$. aureus, and has been effective in animal models of IE caused by this bacterium [43,44]. Anecdotal reports of clinical success with the use oral minocyline in the treatment $S$. aureus IE in humans further support its consideration in future investigations $[45,46]$. The report by Schein et al [17] describing single sulfa therapy (i.e. sulphonamide) for IE has only historical relevance, as this therapy is no longer available for oral use. Trimethroprim - sulfamethoxazole, however, a more contemporary sulfacontaining drug with excellent oral bioavailability has been shown to be inferior to vancomycin in treating uncomplicated right-side S. aureus IE in IVDU when given intravenously [47]. Finally, experience with the use of oral macrolides such as erythromycin in the treatment of severe infections is very limited and the growing rates of resistance to macrolides among streptococci and staphylococci are a further concern [48-50].

Our review has limitations. It is possible that we missed evidence beyond the boundaries of our search strategies. As mentioned above, a majority of the studies included in our analyses had poor methodological quality. The significant heterogeneity in their study populations and designs prevented us from calculating any meaningful pooled estimates. Comprehensive analyses of drug safety, side effects, and comparative costs were largely lacking. We cannot rule out potential publication bias against studies that found poor effectiveness of oral antibiotic therapy in IE. Finally, since we limited our review to studies in which no parenteral antibiotic was used beyond the two weeks of treatment, the value of oral regimens as step-down therapy for IE beyond this time interval might have not been fully captured.

\section{Conclusion}

In conclusion, oral antibiotics with favourable pharmacokinetic profiles appear effective in treating selected cases of IE caused by susceptible organisms. Because of its favourable pharmacokinetic profile, high-dose oral amoxicillin for the treatment of IE caused by susceptible streptococci is particularly appealing but studies of better quality are needed before further recommendations can be made about this approach in clinical settings. The same considerations apply to the use of oral linezolid in cases of $S$. aureus IE. Oral combination therapy with ciprofloxacin and rifampin appears to be an acceptable alternative for the treatment of uncomplicated right-side endocarditis caused by susceptible strains of $S$. aureus in IVDUs but until adequate clinical trials are available, this approach should be reserved for special situations in which conventional IV antibiotic therapy is not possible or it is undesirable. Ongoing and future investigations should help to better define the role of oral antibiotics in the treatment of IE [51].

\section{Additional files}

Additional file 1: Search strategy to identify studies of oral antibiotic therapy in infective endocarditis.

Additional file 2: Articles initially considered for analysis but ultimately excluded based on eligibility criteria.

\section{Abbreviations}

IE: Infective endocarditis; IV: Intravenous; IVDU: Intravenous drug user MIC: Minimum inhibitory concentration.

\section{Competing interests}

The authors have no financial or non-financial competing interests to declare.

\section{Authors' contributions}

Conception of the review: AA-O and VFC-M. Design of the methodology of the review: $\mathrm{AA}-\mathrm{O}, \mathrm{CL}$, DWC and VFC-M. Critical review and interpretation of selected literature: AA-O, CL, DWC and VFC-M. Writing of the initial draft: AA-O. Critical review and editing of the manuscript: AA-O, CL, DWC, and VFC-M. All authors take responsibility for the integrity of the work. All authors read and approved the final manuscript.

\section{Acknowledgements}

We acknowledge Mrs. Alexandra Davis, librarian at the Ottawa Hospital, for her assistance in the design of our search strategies and the retrieval of selected articles. We also acknowledge Dr. Y. Schreiber, from the Division of Infectious Diseases at The Ottawa Hospital, for her assistance with the translation of papers from the French language.

\section{Funding}

VFC-M is supported by a Research Priority Grant from the Department of Medicine of the The Ottawa Hospital, and a Junior Investigator Award of the Ottawa Hospital Research Institute. DWC is supported by a Career Scientist Award of the Ontario Ministry of Health/Ontario HIV Treatment Network. There was no specific funding for this manuscript. No funding bodies had any role in study design, data collection and analysis, decision to publish, or preparation of the manuscript.

\section{Author details}

${ }^{1}$ Department of Medicine, Security Forces Hospital, Riyadh, Saudi Arabia. ${ }^{2}$ Department of Medicine, The Ottawa Hospital, Ottawa, Ontario, Canada. ${ }^{3}$ The Ottawa Hospital Research Institute, Ottawa, Ontario, Canada. ${ }^{4}$ The Department of Medicine, University of Ottawa, Ottawa, Ontario, Canada. ${ }^{5} 1053$ Carling Ave, ASB 2003, Ottawa, Ontario K1Y 4E9, Canada.

Received: 29 October 2013 Accepted: 13 February 2014

Published: 13 March 2014 


\section{References}

1. Mylonakis E, Calderwood SB: Infective endocarditis in adults. N Engl J Med 2001, 345(18):1318-1330

2. Que YA, Moreillon P: Infective endocarditis. Nat Rev Cardiol 2011, 8(6):322-336.

3. Wilson WR, Gilbert DN, Bisno AL, Freedman LR, Smith C, Drusano G, Kaye D: Evaluation of new anti-infective drugs for the treatment of infective endocarditis. Infectious Diseases Society of America and the Food and Drug Administration. Clin Infect Dis 1992, 15 Suppl 1:S89-95.

4. Cunha BA: Oral antibiotic therapy of serious systemic infections. Med Clin North Am 2006, 90(6):1197-1222.

5. Baddour LM, Wilson WR, Bayer AS, Fowler VG Jr, Bolger AF, Levison ME, Ferrieri P, Gerber MA, Tani LY, Gewitz MH, Tong DC, Steckelberg JM, Baltimore RS, Shulman ST, Burns JC, Falace DA, Newburger JW, Pallasch TJ, Takahashi M, Taubert KA, Committee on Rheumatic Fever, Endocarditis, and Kawasaki Disease, Council on Cardiovascular Disease in the Young, Councils on Clinical Cardiology, Stroke, and Cardiovascular Surgery and Anesthesia, American Heart Association, Infectious Diseases Society of America: Infective endocarditis: diagnosis, antimicrobial therapy, and management of complications: a statement for healthcare professionals from the Committee on Rheumatic Fever, Endocarditis, and Kawasaki Disease, Council on Cardiovascular Disease in the Young, and the Councils on Clinical Cardiology, Stroke, and Cardiovascular Surgery and Anesthesia, American Heart Association: endorsed by the Infectious Diseases Society of America. Circulation 2005, 111(23):e394-434.

6. Moher D, Liberati A, Tetzlaff J, Altman DG: Preferred reporting items for systematic reviews and meta-analyses: the PRISMA statement. Ann Intern Med 2009, 151(4):264-269. W264.

7. How to read clinical journals: III. To learn the clinical course and prognosis of disease. Can Med Assoc J 1981, 124(7):869-872.

8. Moher D, Schulz KF, Altman DG: The CONSORT statement: revised recommendations for improving the quality of reports of parallel-group randomised trials. Lancet 2001, 357(9263):1191-1194.

9. Colli A, Campodonico R, Gherli T: Early switch from vancomycin to oral linezolid for treatment of gram-positive heart valve endocarditis. Ann Thorac Surg 2007, 84(1):87-91.

10. Dworkin RJ, Lee BL, Sande MA, Chambers HF: Treatment of right-sided Staphylococcus aureus endocarditis in intravenous drug users with ciprofloxacin and rifampicin. Lancet 1989, 2(8671):1071-1073.

11. Chetty S, Mitha AS: High-dose oral amoxycillin in the treatment of infective endocarditis. S Afr Med J 1988, 73(12):709-710.

12. Pinchas A, Lessing J, Siegman-Igra Y, Liron M: Oral treatment of bacterial endocarditis. Isr J Med Sci 1983, 19(7):646-648.

13. Phillips $B$, Watson GH: Oral treatment of subacute bacterial endocarditis in children. Arch Dis Child 1977, 52(3):235-237.

14. Gray IR, Tai AR, Wallace JG, Calder JH: Oral treatment of bacterial endocarditis with penicillins. Lancet 1964, 2(7351):110-114.

15. Campeau L, Lefebvre M: Oral treatment of malignant streptococcal endocarditis by potassium phenethicillin: 10 cases. Union Med Can 1963 92:873-880

16. Friedberg CK: Treatment of subacute bacterial endocarditis with aureomycin. J Am Med Assoc 1952, 148(2):98-103.

17. Schein J, Baehr G: Sulfonamide therapy of subacute bacterial endocarditis. Am J Med 1948, 4(1):66-72.

18. Heldman AW, Hartert TV, Ray SC, Daoud EG, Kowalski TE, Pompili VJ, Sisson SD, Tidmore WC, Vom Eigen KA, Goodman SN, Lietman PS, Petty BG, Flexner C: Oral antibiotic treatment of right-sided staphylococcal endocarditis in injection drug users: prospective randomized comparison with parenteral therapy. Am J Med 1996, 101(1):68-76.

19. Stamboulian D, Bonvehi P, Arevalo C, Bologna R, Cassetti I, Scilingo V, Efron E: Antibiotic management of outpatients with endocarditis due to penicillin-susceptible streptococci. Rev Infect Dis 1991, 13(Suppl 2):S160-163.

20. Lafforgue G, Arellano C, Vachoux C, Woodley J, Philibert C, Dupouy V, Bousquet-Melou A, Gandia P, Houin G: Oral absorption of ampicillin: role of paracellular route vs. PepT1 transporter. Fundam Clin Pharmacol 2008, 22(2):189-201

21. Gordon C, Regamey C, Kirby WM: Comparative clinical pharmacology of amoxicillin and ampicillin administered orally. Antimicrob Agents Chemother 1972, 1(6):504-507.

22. Sutherland R, Croydon EA, Rolinson GN: Amoxycillin: a new semi-synthetic penicillin. Br Med J 1972, 3(5817):13-16.
23. Gray IR: The choice of antibiotic for treating infective endocarditis. Q J Med 1975, 44(175):449-458.

24. Moreno RA, Boldrina L, Guermani A, Mazucheli J, Sverdloff C, Borges NC: Comparative bioavailability study of two phenoxymethylpenicillin potassium tablet formulations in healthy volunteers. Int $J$ Clin Pharmacol Ther 2007, 45(12):669-676.

25. Watanakunakorn C: The use of beta-lactam antibiotics in the treatment of septicaemia and endocarditis. Scand J Infect Dis Supp/ 1984, 42:110-116.

26. Sabath LD: Phenoxymethylpenicillin (penicillin V) and phenethicillin. Med Clin North Am 1970, 54(5):1101-1111.

27. Bolon MK: The newer fluoroquinolones. Infect Dis Clin North Am 2009, 23 (4):1027-1051. X.

28. Kaatz GW, Barriere SL, Schaberg DR, Fekety R: The emergence of resistance to ciprofloxacin during treatment of experimental Staphylococcus aureus endocarditis. J Antimicrob Chemother 1987, 20(5):753-758.

29. Zak O, Scheld WM, Sande MA: Rifampin in experimental endocarditis due to Staphylococcus aureus in rabbits. Rev Infect Dis 1983, 5(Suppl 3):S481-490

30. Hackbarth CJ, Chambers HF, Sande MA: Serum bactericidal activity of rifampin in combination with other antimicrobial agents against Staphylococcus aureus. Antimicrob Agents Chemother 1986, 29(4):611-613

31. Kaatz GW, Seo SM, Barriere SL, Albrecht LM, Rybak MJ: Ciprofloxacin and rifampin, alone and in combination, for therapy of experimental Staphylococcus aureus endocarditis. Antimicrob Agents Chemother 1989, 33(8):1184-1187.

32. Tebas P, Martinez Ruiz R, Roman F, Mendaza P, Rodriguez Diaz JC, Daza R, de Letona JM: Early resistance to rifampin and ciprofloxacin in the treatment of right-sided Staphylococcus aureus endocarditis. I Infect Dis 1991, 163(1):204-205.

33. Dajcs JJ, Thibodeaux BA, Marquart ME, Girgis DO, Traidej M, O'Callaghan RJ: Effectiveness of ciprofloxacin, levofloxacin, or moxifloxacin for treatment of experimental Staphylococcus aureus keratitis. Antimicrob Agents Chemother 2004, 48(6):1948-1952.

34. Lister PD: Pharmacodynamics of moxifloxacin and levofloxacin against Staphylococcus aureus and Staphylococcus epidermidis in an in vitro pharmacodynamic model. Clin Infect Dis 2001, 32(Suppl 1):S33-38.

35. Entenza JM, Vouillamoz J, Glauser MP, Moreillon P: Levofloxacin versus ciprofloxacin, flucloxacillin, or vancomycin for treatment of experimental endocarditis due to methicillin-susceptible or -resistant Staphylococcus aureus. Antimicrob Agents Chemother 1997, 41(8):1662-1667.

36. Galani L, Pefanis A, Sakka V, lliopoulos D, Donta I, Triantafyllidi H, Skiadas I, Karayiannakos P, Giamarellou H: Successful treatment with moxifloxacin of experimental aortic valve endocarditis due to methicillin-resistant Staphylococcus aureus (MRSA). Int J Antimicrob Agents 2009, 33(1):65-69.

37. Berrington AW, Koerner RJ, Perry JD, Bain HH, Gould FK: Treatment of Staphylococcus aureus endocarditis using moxifloxacin. Int J Med Microbiol 2001, 291(3):237-239.

38. Foo SS, Lye DC: Methicillin-sensitive Staphylococcus aureus tricuspid valve endocarditis with annular abscess cured with oral levofloxacin and rifampicin. Int J Antimicrob Agents 2007, 30(3):275-277.

39. Dryden MS: Linezolid pharmacokinetics and pharmacodynamics in clinical treatment. J Antimicrob Chemother, 66 Suppl 4:iv7-iv15.

40. Hall B: The absorption and distribution of aureomycin in man; a review of the literature and study of the concentration of aureomycin in the serum, urine and cerebrospinal fluid after oral administration. Ann Intern Med 1954, 40(4):743-754

41. Rolain JM, Boulos A, Mallet MN, Raoult D: Correlation between ratio of serum doxycycline concentration to MIC and rapid decline of antibody levels during treatment of $\mathrm{Q}$ fever endocarditis. Antimicrob Agents Chemother 2005, 49(7):2673-2676.

42. Rolain JM, Mallet MN, Raoult D: Correlation between serum doxycycline concentrations and serologic evolution in patients with Coxiella burnetii endocarditis. J Infect Dis 2003, 188(9):1322-1325.

43. Cunha BA: Minocycline versus doxycycline for meticillin-resistant Staphylococcus aureus (MRSA): in vitro susceptibility versus in vivo effectiveness. Int J Antimicrob Agents 2010, 35(5):517-518.

44. Nicolau DP, Freeman CD, Nightingale CH, Coe CJ, Quintiliani R: Minocycline versus vancomycin for treatment of experimental endocarditis caused by oxacillin-resistant Staphylococcus aureus. Antimicrob Agents Chemother 1994, 38(7):1515-1518. 
45. Clumeck N, Marcelis L, Amiri-Lamraski MH, Gordts B: Treatment of severe staphylococcal infections with a rifampicin-minocycline association. J Antimicrob Chemother 1984, 13 Suppl C:17-22.

46. Lawlor MT, Sullivan MC, Levitz RE, Quintiliani R, Nightingale C: Treatment of prosthetic valve endocarditis due to methicillin-resistant Staphylococcus aureus with minocycline. J Infect Dis 1990, 161(4):812-814.

47. Markowitz N, Quinn EL, Saravolatz LD: Trimethoprim-sulfamethoxazole compared with vancomycin for the treatment of Staphylococcus aureus infection. Ann Intern Med 1992, 117(5):390-398.

48. Ono T, Shiota S, Hirota K, Nemoto K, Tsuchiya T, Miyake Y: Susceptibilities of oral and nasal isolates of Streptococcus mitis and Streptococcus oralis to macrolides and PCR detection of resistance genes. Antimicrob Agents Chemother 2000, 44(4):1078-1080.

49. McKenna S, Evans G: Macrolides: A Canadian Infectious Disease Society position paper. Can J Infect Dis 2001, 12(4):218-231.

50. Prunier AL, Malbruny B, Tande D, Picard B, Leclerca R: Clinical isolates of Staphylococcus aureus with ribosomal mutations conferring resistance to macrolides. Antimicrob Agents Chemother 2002, 46(9):3054-3056.

51. Iversen K, Host N, Bruun NE, Elming H, Pump B, Christensen JJ, Gill S, Rosenvinge F, Wiggers H, Fuursted K, Holst-Hansen C, Korup E, Schønheyder HC, Hassager C, Høfsten D, Larsen JH, Moser C, Ihlemann N, Bundgaard H: Partial oral treatment of endocarditis. Am Heart J 2013, 165(2):116-122.

doi:10.1186/1471-2334-14-140

Cite this article as: Al-Omari et al:: Oral antibiotic therapy for the treatment of infective endocarditis: a systematic review. BMC Infectious Diseases 2014 14:140.

\section{Submit your next manuscript to BioMed Central and take full advantage of:}

- Convenient online submission

- Thorough peer review

- No space constraints or color figure charges

- Immediate publication on acceptance

- Inclusion in PubMed, CAS, Scopus and Google Scholar

- Research which is freely available for redistribution 